\title{
ON THE CLASSIFICATION OF TAUT SUBMANIFOLDS
}

\author{
BY MICHAEL FREEDMAN
}

Communicated by Samuel Eilenberg, April 28, 1975

All terminology will be smooth. A submanifold $K^{2 n} \stackrel{i}{\longrightarrow} M^{2 n+2}$ is taut if $\pi_{i}(U, \partial U)=0$ for $i \leqslant n$, where $U=(M$-neighborhood $K)$. Examples are: nonsingular algebraic hypersurfaces in $C P^{n}$ (this follows from the Lefschetz theorem on hyperplane sections), simple knots (see $[\mathbf{L}]$ ), the spines (see $[\mathbf{M}]$ ). Every codimension-2 homology class contains taut representatives (see [K-M]), and the set of taut submanifolds is closed under connected sum (of pairs) with ( $S^{n} \times S^{n}$ $\stackrel{\text { standard }}{\longrightarrow} S^{2 n+2}$ ). Taut submanifolds are "almost canonical" in the sense of [Q], and from this viewpoint it is readily seen that if $n \geqslant 3$, every $K^{2 n C i} \longrightarrow M^{2 n+2}$ with $i n$-connected is concordant to $K^{2 n} \stackrel{i^{\prime}}{\longleftrightarrow} M^{2 n+2}$ taut.

If $M^{2 n+2}$ is simply connected, the homology groups of $K^{2 n}$, taut, are completely determined by the homology of $M^{2 n+2}$ except for $B_{n}\left(K^{2 n}\right)$. A lower bound on $B_{n}(K)$ in terms of $i_{*}\left[K^{2 n}\right]$ and the cohomology ring of $M^{2 n+2}$ has been obtained in [T-W]. In [F 1] we have proven Theorem 1 , which provides a partial converse to Theorem 2.2 of [T-W] for $M \cong C P^{n+1}, n>2$ odd, and $i_{*}[K]=$ a prime, $p$, multiple of the generator of $H_{2 n}\left(C P^{n+1} ; Z\right)$. Interestingly, if $p>3$, the nonsingular algebraic hypersurfaces $V$ are not the simplest taut submanifolds in their homology class, but may be decomposed as $V=K \#_{l \text {-copies }}$ $S^{n} \times S^{n}, l>0$, for some taut submanifold $K$.

We do not know if this is true for $n=1$. If it were, there would be surfaces imbedded in $C P^{2}$ with genus smaller than that of the nonsingular algebraic hypersurfaces to which they are homologous. This would contradict Thom's conjecture.

Statement of Theorem 1. Let $M^{2 n+2}$ be a simply-connected, oriented, smooth $(2 n+2)$-manifold, $n$ odd $>1$. Let $x \in H^{2}\left(M^{2 n+2} ; Z\right)$ generate a free summand of $H^{2}\left(M^{2 n+2} ; Z\right)$. Let $p$ be any prime. Set

$$
\begin{aligned}
& \bar{s}_{\text {even }}=\max \{4,(\cosh (p-2 k) x)(\operatorname{sech}(p x))(L(M))[M] \mid 0<k<p\}, \\
& \bar{s}_{\text {odd }}=\max \{3,(\cosh (p-2 k) x)(\operatorname{sech}(p x))(L(M))[M] \mid 0<k<p\},
\end{aligned}
$$

where $L$ is the Hirzebruch polynomial.

For all integers $h \geqslant 0$, there exists a taut submanifold $K_{h} \stackrel{i}{\longrightarrow} M$ with

$$
M \cap p x=i_{*}\left[K_{h}\right],
$$

AMS (MOS) subject classifications (1970). Primary 57D95; Secondary 57D65. 
and

$$
\begin{array}{r}
B_{n}\left(K_{h}\right)=\bar{s}_{\text {even }}+6 T_{n}(M)-2 B_{n}(M)+B_{n+1}(M)+2 h \\
\text { if } B_{n+1}(M) \text { is even. } \\
=\bar{s}_{\text {odd }}+6 T_{n}(M)-2 B_{n}(M)+B_{n+1}(M)+2 h \\
\text { if } B_{n+1}(M) \text { is odd }
\end{array}
$$

$B_{n}(M)=\operatorname{rank} H_{n}(M ; Z) /$ Torsion, $T_{n}(M)=\operatorname{rank} H_{n}(M)=\operatorname{rank} H_{n}(M ; Z)$.

We now state two theorems, proved in [F2], which indicate to what extent the diffeomorphism class of a taut submanifold is fixed by $B_{n}(K)$.

THEOREM 2. If $M^{2 n+2}$ is a compact, simply connected, smooth $(2 n+2)$ manifold, $n$ odd $\geqslant 3$, and $K_{0}^{2 n} \stackrel{i_{0}}{\longrightarrow} M^{2 n+2}$ and $K_{1}^{2 n} \stackrel{i_{1}}{\longrightarrow} M^{2 n+2}$ are $n$ connected inclusions of closed submanifolds with $\left(i_{0}\right)_{*}\left[K_{0}\right]=\left(i_{1}\right)_{*}\left[K_{1}\right] \in$ $H_{2 n}\left(M^{2 n+2} ; Z\right)$, then if $B_{n}\left(K_{0}\right)=B_{n}\left(K_{1}\right), K_{0}$ is diffeomorphic to $K_{1}$.

Theorem 3. Assume $M^{2 n+2}$ is a simply-connected smooth $(2 n+2)$-manifold, $n$ even, $\geqslant 2$, with $H_{n}(M ; Z)=0$. If $i_{0}$ and $i_{1}$ are as above, then if the intersection pairings on $H_{n}\left(K_{0} ; Z\right) /$ Torsion and $H_{n}\left(K_{1} ; Z\right) /$ Torsion are isometric, $K_{0}$ is diffeomorphic to $K_{1}$.

If $M^{2 n+2}, n$ odd, $\geqslant 3$, is simply-connected, it follows from Theorem 2 that there is a simplest taut submanifold representing $i_{*}[K], K_{0}$, and every other is of the form $K_{l}=K_{0} \#_{l \text {-copies }} S^{n} \times S^{n}$. This, together with a previous remark, yields a complete classification of taut submanifolds in a homotopy $C P^{n+1}, n$ odd, $\geqslant 1$, representing a prime multiple of the generator of $H_{2 n}\left(C P^{n+1} ; Z\right)$.

\section{REFERENCES}

[F 1$]$ M. Freedman, Surgery on codimension-2 submanifolds (to appear).

[F2] Uniqueness theorems for taut submanifolds (to appear).

[K-M] M. Kato and Y. Matsumoto, Simply connected surgery of submanifolds in codimension two. I, J. Math. Soc. Japan 24 (1972), 586-608. MR 46 \#6369.

[Q] F. Quinn, Almost canonical inverse images, Comment. Math. Helv. (1974).

[L] J. Levine, Knot cobordism groups in codimension two, Comment. Math. Helv. 44 (1969), 229-244. MR 39 \#7618.

[M] Y. Matsumoto, Knot cobordism and surgery in codimension 2, J. Fac. Sci. Univ. Tokyo Sect. IA 20 (1973), 253-317.

DEPARTMENT OF MATHEMATICS, UNIVERSITY OF CALIFORNIA, BERKELEY, CALIFORNIA 94720 